Nous ouvrons ici une nouvelle rubrique intitulée : "Comptes rendus d'actions thématiques ».

Ces actions sont entreprises sur programme d'Instituts de Recherche

Le Comité Scientifique de Le Lait a jugé utile de publier de tels comptes rendus bien que, dans leur formulation et leur présentation, ils soient éloignés des publications scientifiques habituelles. En effet, ils réunissent résultats et conclusions en un ensemble synthétique et élaboré.

\title{
Maîtrise de l'affinage des fromages de type Camembert
}

\author{
Compte rendu d'une Action Thématique programmée \\ conduite du 1-8-80 au 31-8-83
}

Institut National Agronomique, C.N.R.Z. - 78350 Jouy-en-Josas (France)

(B. RIBADEAU-DUMAS, coordonnateur)

\section{S u m m a r y}

Studies ON THE CONTROL OF THE RIPENING OF CAMEMBERT TYPE CHEESES

This is a report on a collaborative study grouping several French laboratories and firms under the leadership of the Institut National de la Recherche Agronomique. The first chapter gives the aim of the research (better reliability, possible modifications for obtaining desired flavor and texture) and the list of participants. The second chapter details the procedure employed for the organoleptic analyses, while the third one presents the main results. Conclusions are exposed in the 4th chapter. The 5th one gives the list of publications and reports which have been produced during the 3 year investigations.

Good reliability was obtained by using pasteurized milk, one strain of lactic bacteria (S. cremoris), one strain of Penicillium 
caseicolum and a technology which reduced microbial contaminations. However this invariably led to a high level of bitterness and a lack of typical camembert flavor. It was shown that the amount of rennet had negligible influence on bitterness. This defect was remarkably reduced by incubating the cheeses in ammonia atmosphere during the first days of ripening. This caused a much slower development of the mold and reduced the action of its acids proteinase, which was thus supposed to be responsible for the release of bitter peptides. It was shown that some strains of Geotrichum candidum, used together with Penicillium, had similar effects in reducing Penicillium growth, acid proteinase action and bitterness. Furthermore Geotrichum gave a "sulphur» flavor which is typical of traditional Camembert cheese.

The influence of $p H$ on the texture modifications which occurs during ripening was demonstrated by experiments in ammonia atmosphere. Furthermore, the migration of calcium and phosphate from the inside to the outside of cheese, previously attributed to some "sequestring power» of Penicillium, was shown to be $\mathrm{pH}$ dependent.

The evolution with ripening time of the microbial flora of traditional Camembert cheeses was investigated in different firms. Special attention was paid to microorganisms such as B.linens, that are somewhat specific of this type of cheese. Organoleptic comparisons were carried out between experimental cheeses and commercial products from different origins. The surface flora was studied during ripening by scanning electron microscopy.

\section{Ré s u m é}

Cet article est un résumé des travaux correspondant à une Action Thématique Programmée (A.T.P.) réalisée sous l'égide de l'Institut National Agronomique. Le premier chapitre expose l'objet des recherches (obtention de fabrications plus régulières, des caractéristiques recherchées en ce qui concerne la flaveur et la texture) et la liste des participants. Le second chapitre détaille les techniques employées pour les analyses organoleptiques, et le $3^{\text {e }}$ présente les résultats principaux. Les conclusions sont rassemblées dans le $4^{\mathrm{e}}$ chapitre. Le $5^{\mathrm{e}}$ et dernier chapitre donne la liste des publications et rapports auxquels a donné lieu l'A.T.P.

Une bonne reproductibilité dans les fabrications a été obtenue en utilisant du lait pasteurisé, une souche de ferment lactique (S. cremoris), une souche de P.caseicolum et une technologie qui réduisent les contaminations microbiennes. Cependant ceci a conduit invariablement à des produits possédant une amertume intense et 
manquant des caractéristiques organoleptiques typiques du Camembert traditionnel. Il a été montré que la quantité de présure utilisée avait une influence négligeable sur le degré d'amertume. Par contre, ce défaut a été considérablement réduit par incubation des fromages en atmosphère ammoniacale pendant les premiers jours d'affinage. Ceci conduisait à un développement beaucoup plus lent de la moisissure et réduisait l'action de la protéase acide qu'elle produit. Il semble ainsi que cette enzyme soit responsable de la libération de peptides de goût amer. On a montré que certaines souches de Geotrichum candidum, utilisées avec $P$. caseicolum, avaient un effet similaire, réduisant la croissance du Penicillium, l'action de la protéase acide et le degré d'amertume. De plus, le Geotrichum donnait une saveur "soufrée " typique des Camemberts traditionnels. L'influence $\mathrm{du} \mathrm{pH}$ sur les modifications de texture qui apparaissent durant l'affinage a été démontrée par des essais effectués en atmosphère ammoniacale. On a montré que la migration du calcium et du phosphate, de l'intérieur vers l'extérieur du fromage, attribuée auparavant à un " pouvoir séquestrant " du Penicillium, dépendait en fait du $\mathrm{pH}$.

L'évolution de la flore microbienne pendant l'affinage du Camembert traditionnel a été suivie dans différents ateliers normands. Une attention spéciale a été portée à des micro-organismes tels que $B$. linens, qui sont dans une certaine mesure caractéristiques des fromages traditionnels. Des comparaisons organoleptiques ont été faites entre fromages expérimentaux et fromages commerciaux de différentes origines. La flore de surface a été étudiée durant l'affinage par microscopie électronique à balayage.

Une "Commission Lait » de l'I.N.R.A., qui regroupait industriels, chercheurs et représentants de l'Administration, s'est réunie périodiquement de 1975 à 1979, sous la présidence de J. Auclair, afin d'étudier l'évolution prévisible du secteur laitier français à moyen et long terme. Elle a, à la fin de ses travaux, proposé aux chercheurs du "Secteur Lait » de l'I.N.R.A. trois thèmes de recherches prioritaires concernant la Recherche Laitière en France. La « maîtrise de l'affinage " des fromages était l'un d'entre eux et, en 1979, la Direction Scientifique du Secteur des Industries Agro-Alimentaires de I'I.N.R.A. lançait une action thématique (A.T.P.) sur ce sujet, le fromage choisi pour l'étude étant le Camembert. Il nous a semblé utile de rendre publics les principaux résultats obtenus durant les trois années d'expérimentation.

Dans un premier chapitre seront rappelés les objectifs initiaux et la liste des participants de l'A.T.P.

Il nous a également semblé utile d'exposer ensuite (chap. II) en détail le protocole des analyses organoleptiques pratiquées à Jouy- 
en-Josas. Pour les autres techniques analytiques utilisées (biochimiques, microbiologiques, rhéologiques, biométriques) et le protocole de fabrication à Jouy-en-Josas, le lecteur pourra se référer aux publications issues de l'A.T.P.

Le $3^{e}$ chapitre fera état des principaux résultats qui ont été obtenus. Les conclusions en seront tirées dans le $4^{\mathrm{e}}$ chapitre. Enfin, la liste des mémoires et publications auxquels ont donné lieu le travail accompli constitue le $5^{\mathrm{e}}$ chapitre.

\section{Chapitre I : OBJECTIFS ET PARTICIPANTS}

\section{I.1. Objectifs}

L'objectif général était celui qui a été défini par la " Commission Lait ». Cet objectif comprenait deux volets qui sont liés :

- maîtrise de l'affinage ;

- connaissance des composés d'arôme;

le fromage choisi pour l'étude étant le Camembert.

Maîtriser l'affinage, cela signifie être en mesure d'assurer des fabrications aussi régulières que possible, de modifier en connaissance de cause certains paramètres technologiques ou microbiologiques de façon à obtenir un élément désiré de flaveur ou de texture.

Ceci implique donc des techniques fiables permettant d'apprécier cette flaveur ou cette texture; ceci implique également la connaissance des mécanismes de production des composés d'arôme, de constitution de la texture.

Dans cette optique, les objectifs de l'A.T.P. ont été décrits d'une manière plus précise comme suit :

1. Mise en place au L.B.T.L. d'un jury de dégustation composé de membres des 3 laboratoires " lait » de Jouy (Arômes, L.B.T.L.*, L.M.L.G.A.) et avec le concours du Département de Biométrie.

Le jury, composé de 30 personnes, fonctionnait régulièrement depuis quelques mois. Il avait pour missions :

- par la dégustation régulière de Camemberts affinés du commerce, de mettre au point un vocabulaire aussi précis que possible permettant de caractériser et de quantifier les différentes composantes de l'arôme et de la texture;

* Voir la signification des abréviations dans la liste des participants. 
- par la dégustation de Camemberts affinés fabriqués régulièrement à la Laiterie Expérimentale de Jouy, d'apprécier la régularité des fabrications au plan organoleptique;

- de permettre la mise en évidence, par la suite, de l'influence de tel ou tel paramètre physique, chimique, microbiologique ou technologique sur les caractéristiques organoleptiques. En d'autres termes, le but final était de substituer autant que possible à l'appréciation organoleptique une appréciation analytique plus objective lorsque des corrélations nettes auraient été trouvées entre certains caractères organoleptiques et certaines caractéristiques biochimiques mesurables.

Le jury de dégustation de Jouy pouvait travailler en relation avec ceux du Centre de Recherches B.E.L. et de l'E.N.S.I.A.

2. Mise en place à la Laiterie Expérimentale de Jouy, de fabrications aussi régulières que possible.

Les irrégularités de fabrication, qui constituent l'un des problèmes les plus importants de la fromagerie française, et même internationale, peuvent provenir du lait, des micro-organismes et de la technologie. Il semble que les micro-organismes (et les bactériophages) en soient la cause principale. Relier tel ou tel paramètre analytique du fromage à telle ou telle caractéristique organoleptique requiert en premier lieu l'appréciation de l'étendue des variations de ces paramètres dans les conditions de fabrication choisies. Si le lait et la technologie peuvent être plus ou moins facilement contrôlés, il n'en n'est pas de même du développement des microorganismes. Le protocole retenu s'éloignait sensiblement de la pratique industrielle, en ce sens qu'il tendait à rendre négligeables les contaminations microbiennes et le développement des phages par des conditions d'asepsie aussi rigoureuses que possible.

Les fabrications réalisées à Jouy devaient être soumises à des analyses poussées d'ordres rhéologique, biochimique, physicochimique et organoleptique de la part des différents laboratoires participant à l'A.T.P., en relation avec le Département de Biométrie de I'I.N.R.A.

3. Etude des modifications organoleptiques et analytiques produites dans ces fabrications par des modifications ponctuelles d'ordre microbiologique ou technologique.

\section{I.2. Participants}

\subsubsection{Participants extérieurs à l'I.N.R.A.}

- Syndicat des Fabricants du Véritable Camembert de Normandie (S.V.C.N.)

8 , rue de Bernières, Caen

Secrétaire Général : M. R. Michel-Bechet. 
- Centre de Recherches des Fromageries B.E.L. Boulevard de l'Industrie, 41 Vendôme Responsable A.T.P. : M. G. Delespaul.

- Ecole Nationale Supérieure des Industries Agricoles et Alimentaires (E.N.S.I.A.)

Laboratoire de Rhéologie Appliquée aux I.A.A.

1 , avenue des Olympiades - 91305 Massy

M. B. Launay, professeur.

\subsubsection{Participants I.N.R.A.}

- Laboratoire d'Etude des Arômes, M. J. Adda, Directeur, C.N.R.Z. 78350 Jouy-en-Josas.

- Laboratoire de Biochimie et Technologie Laitières (L.B.T.L.), M. B. Ribadeau-Dumas, Directeur - 78350 Jouy-en-Josas.

- Laboratoire de Microbiologie Laitière et Génie Alimentaire (L.M.L.G.A.), M. J.-L. Bergère, Directeur - 78350 Jouy-en-Josas.

- Laboratoire de Biométrie, M. R. Tomassone, Directeur - 78350 Jouy-en-Josas.

- Laboratoire de Recherches de Technologie Laitière (L.R.T.L.), M. J.-L. Maubois, Directeur, Centre de Recherches I.N.R.A. de Rennes, 65, rue de Saint-Brieuc - 35042 Rennes cedex.

- Station Expérimentale Laitière I.N.R.A., M. Rousseaux, Directeur 39800 Poligny.

- Laboratoire de Recherches Fromagères I.N.R.A., M. Devoyod, Directeur - 15000 Aurillac.

- Laboratoire de Technologie, M. J. Lenoir, Directeur, Institut National Agronomique Paris-Grignon, Centre de Grignon - 78850 Thiverval-Grignon.

\section{Chapitre II : ANALYSES ORGANOLEPTIQUES}

Les membres du jury de Jouy ont d'abord été entrainés à reconnaître les saveurs fondamentales (sucré, salé, acide, amer), puis le groupe a procédé à la mise au point d'un vocabulaire commun. Des termes descriptifs de la texture et de l'arôme ont été librement émis par les membres du groupe au cours de dégustations de fromages de la Laiterie de Jouy et de fromages du commerce. Au total, 58 descripteurs ont été recueillis pour la texture, et le même nombre pour l'arôme. Ils ont été regroupés en un nombre de classes plus restreint (7 pour la texture, et 14 pour l'arôme) en faisant appel à des analyses de correspondance et de classification automatique effectuées par le Laboratoire de Biométrie. A partir de ces résultats, des fiches de dégustation ont été mises au point par les membres du groupe de façon à tendre vers une unité de langage. 
La quantification de l'intensité des caractères ainsi définis était effectuée sur une échelle structurée à 5 niveaux. L'interprétation des résultats était effectuée par analyse de variance, au moyen des programmes du Laboratoire de Biométrie, selon les principes de l'analyse descriptive quantitative. Cette méthode a été choisie en raison de sa robustesse, malgré son incompatibilité théorique avec le type de données recueillies.

Parallèlement à ces analyses descriptives, le jury a procédé à de nombreuses comparaisons d'échantillons faisant appel aux épreuves de classement classiques (épreuves par paires, triangulaires, etc.).

Sont données dans ce qui suit :

1. La description des termes utilisés pour décrire la texture.

2. L'échelle de quantification des caractères étudiés dans les deux fiches suivantes.

3. La fiche donnée au dégustateur pour une analyse de texture.

4. La fiche donnée au dégustateur pour une analyse de goût.

5. La fiche donnée au dégustateur pour la comparaison de deux échantillons à un témoin.

Le jury comprenait au total 25 personnes. Chaque dégustation était effectuée par 18 personnes, réparties en 3 groupes de 6 (le nombre de place de la salle de dégustation est de 6).

Des dégustations réalisées selon un protocole différent ont été également effectuées par le Centre de Recherches B.E.L.

\section{Chapitre III : PRINCIPAUX RESULTATS}

\section{III.1. Jury de dégustation}

Le jury a fonctionné d'une façon tout à fait satisfaisante durant l'A.T.P., et ses activités se poursuivent toujours.

La technique d'analyse descriptive quantitative utilisée a permis de distinguer avec des probabilités élevées $(\mathrm{P}<0,01)$ par quels caractères de texture et de flaveur les fromages expérimentaux fabriqués à Jouy (à partir de lait pasteurisé) se distinguaient de fromages normands de lait cru et de fromages ensemencés avec différents micro-organismes (levures, moisissures). Les résultats concernant l'utilisation de Geotrichium candidum (pub. 16, 24), dans le cadre d'un travail effectué par le L.M.L.G.A., auquel B.E.L. a également apporté sa contribution, sont particulièrement significatifs à cet égard.

De même, le jury a pu mettre en évidence de façon hautement significative les différences d'intensité d'amertume de fromages fabri- 


\section{DESCRIPTION DES TERMES UTILISES POUR DECRIRE} LA TEXTURE

\section{Définition}

Mou : par opposition à " dur ».

Dur : qui offre une résistance à la mastication. Souple: qui supporte sans se rompre une certaine déformation.

Friable : qui se désagrège sous l'effort de mastication.

Granuleux : qui présente des petits grains plus ou moins durs.

Onctueux : homogène quant à la structure de la pâte.

Rêche : qui donne l'impression de présenter des aspérités et s'avale difficilement.

Glissant : par opposition à rêche, qui s'avale facilement.
Synonymes ou adjectifs

qui indiquent une différence

d'intensité mais non de nature

Tendre.

Ferme.

Elastique, caoutchouteux, compact.

Cassant.

Farineux, sableux, grumeleux.

Moelleux, fondant, pâteux, collant.

Râpeux, rugueux, sec, étouffant.

Fluide, "gras », lisse.

Echelle utilisée pour l'appréciation quantitative de la texture et du goût :

0 caractère absent (non reconnu).

1 caractère reconnu - intensité faible.

2 caractère reconnu - intensité moyenne.

3 caractère reconnu - intensité forte.

4 caractère reconnu - intensité très forte.

qués avec diverses enzymes coagulantes ou affinées (ou non) en présence d'ammoniac, comme il sera exposé plus loin (pub. 24).

Une bonne corrélation a été observée avec les appréciations du jury en ce qui concerne la fermeté des fromages et les mesures obtenues par le test de pénétrométrie mis au point au L.B.T.L. (pub. 13).

\section{III.2. Mise en place à Jouy de fabrications reproductibles}

Les irrégularités de fabrication constatées industriellement peuvent provenir du lait, de la technologie et surtout des microorganismes présents dans le caillé.

Le protocole de fabrication utilisé à Jouy visait donc d'une part, à limiter au maximum les contaminations, et d'autre part, à assurer une fermentation lactique aussi régulière que possible. Pour ce faire, le lait, provenant d'un troupeau de vaches F.F.P.N. à mises bas synchronisées (ferme I.N.R.A. de Bressonvillers), était pasteurisé peu 


\section{AU DEGUSTATEUR POUR UNE ANALYSE DE STRUCTURE}

Nom :

Date :

1. Aspect

Caractère étudié
Mur (fait)
Plâtreux
Coulant
Croûtage

2. Sensation dans la bouche échantillon $\mathrm{n}^{\circ}$

adjectifs de sens opposé servent à décrire le même caractère.

Ceci a pour but de permettre une description complète des échantillons hétérogènes (affinés sous croûte et plâtreux au centre). Dans le cas où l'échantillon est homogène, il suffit de remplir la ligne correspondant à l'adjectif approprié, l'autre étant automatiquement noté 0 .

\begin{tabular}{l|l} 
Dureté & $\begin{array}{l}\text { mou } \\
\text { dur }\end{array}$ \\
Cohésion & $\begin{array}{l}\text { souple } \\
\text { friable }\end{array}$ \\
$\begin{array}{l}\text { Structure } \\
\text { de la pâte }\end{array}$ & $\begin{array}{l}\text { onctueux } \\
\text { granuleux }\end{array}$ \\
$\begin{array}{l}\text { Sensation } \\
\text { sur la } \\
\text { muqueuse }\end{array}$ & $\begin{array}{l}\text { glissant } \\
\text { rêche }\end{array}$ \\
\hline
\end{tabular}

$\begin{array}{lllll}0 & 1 & 2 & 3 & 4 \\ 0 & 1 & 2 & 3 & 4 \\ 0 & 1 & 2 & 3 & 4 \\ 0 & 1 & 2 & 3 & 4 \\ 0 & 1 & 2 & 3 & 4 \\ 0 & 1 & 2 & 3 & 4 \\ 0 & 1 & 2 & 3 & 4 \\ 0 & 1 & 2 & 3 & 4\end{array}$

de temps avant l'emprésurage (maturation courte) et ensemencé avec un levain lactique de composition connue. Un levain composé d'une seule souche de Streptococcus cremoris, maintenue indemne de phages, a été comparé à des mélanges de 4 souches de streptocoques utilisées en rotation.

La fabrication et l'égouttage étaient effectués dans un local maintenu à température constante. Pendant la première partie de l'A.T.P., le moulage était effectué manuellement. Il a ensuite été réalisé au moyen d'un dispositif Cartier qui permettait d'opérer dans des conditions mieux définies.

Les fromages étaient affinés dans des locaux maintenus en surpression d'air filtré et chaque lot était isolé des autres par des cloches en matière plastique.

La régularité des fabrications a été jugée essentiellement sur les caractères physico-chimiques du caillé égoutté et des fromages 
Nom :

échantillon $n^{\circ}$

\section{Date :}

Caractère étudié : goût (échantillon écroûté) :

$\begin{array}{llllll}\text { 1. Amer } & 0 & 1 & 2 & 3 & 4 \\ \text { 2. Sucré } & 0 & 1 & 2 & 3 & 4 \\ \text { 3. Salé (fromage normalement salé : note 2) } & 0 & 1 & 2 & 3 & 4 \\ \text { 4. Acide, aigre, petit-lait } & 0 & 1 & 2 & 3 & 4 \\ \text { 5. Piquant, acide gras, méthylcétones, caprylique, chèvre } & 0 & 1 & 2 & 3 & 4 \\ \text { 6. Ammoniacal } & 0 & 1 & 2 & 3 & 4 \\ \text { 7. Laitage, lait concentré, caillé frais } & 0 & 1 & 2 & 3 & 4 \\ \text { 8. Champignon, pénicillium, cave, renfermé, paille } & 0 & 1 & 2 & 3 & 4 \\ \text { 9. Soufré, choux, ail } & 0 & 1 & 2 & 3 & 4 \\ \text { 10. Végétal, herbe, foin } & 0 & 1 & 2 & 3 & 4 \\ \text { 11. Floral, fleurie } & 0 & 1 & 2 & 3 & 4 \\ \text { 12. Modification de la graisse (1) } & 0 & 1 & 2 & 3 & 4\end{array}$

12. Modification de la graisse (1)

rance

oxydé métallique

gras

huileux

suiffeux

noisette

13. Fruité

savon

14 Autres

Degré d'affinage $\quad \begin{array}{llllll}0 & 1 & 2 & 3 & 4\end{array}$

$\begin{array}{lllll}0 & 1 & 2 & 3 & 4\end{array}$

$\begin{array}{llllll}\text { Note de qualité } & 0 & 1 & 2 & 3 & 4\end{array}$

très mauvais très bon

après salage $(\mathrm{pH}$, teneur en eau, gras/sec, azote, calcium total, sel) et sur l'importance des contaminations (coliformes, levures, flore non lactique).

Les paramètres les plus importants pour la régularité des fabrications étaient :

a) La nature du levain

En moulage manuel, le $\mathrm{pH}$ moyen des fromages au démoulage, mesuré pour 12 lots de fromages fabriqués avec 4 mélanges de 4 souches utilisées en rotation ( 3 répétitions par souche) et pour 16 fabrications faites avec une seule souche de streptocoque lactique 
Nom :

échantillon $\mathrm{n}^{\circ}$

Date :

et $\mathrm{n}^{\circ}$

\section{COMPARAISON DE DEUX ECHANTILLONS A UN TEMOIN}

Indiquez en plaçant une croix dans la case convenable du tableau ci-dessous si chacun des échantillons $n^{\circ}$ et $n^{\circ}$ est à votre avis :

certainement identique au Témoin peut-être identique au Témoin $\mathrm{T}$ peut-être différent du Témoin T? certainement différent du Témoin $\mathrm{X}$ ? $\mathrm{X}$

\begin{tabular}{c|c|c|c|c}
\hline & $\mathrm{T}$ & $\mathrm{T} ?$ & $\mathrm{X} ?$ & $\mathrm{X}$ \\
\hline & & & & \\
\hline & & & & \\
\hline
\end{tabular}

(C.N.R.Z. 158) était de 4,63 (écart-type 0,1) pour les mélanges et de 4,63 (écart-type 0,07 ) pour la souche pure. Les teneurs en calcium étaient respectivement égales à $0,38 \%$ (écart-type 0,05 ) et $0,34 \%$ (écart-type 0,03 ). Ces résultats ont été obtenus en l'absence de phages homologues des souches utilisées dans le lactosérum de fabrication.

\section{b) Le procédé de fabrication}

Le pH moyen du lait à l'emprésurage a été abaissé de 6,50 à 6,35 lors de l'utilisation du système Cartier en vue d'obtenir un caillé suffisamment ferme pour permettre une utilisation normale du matériel de moulage. Le $\mathrm{pH}$ des fromages au démoulage était plus bas (moyenne : 4,46; écart-type 0,05 ) et la teneur en calcium était corrélativement plus faible (moyenne $0,26 \%$; écart-type 0,01 ) pour les fabrications «Cartier » comparés aux fabrications manuelles.

Après salage, la composition des fromages différait également selon le mode de fabrication, qu'il s'agisse de la composition moyenne ou de la régularité des fabrications. On obtenait respectivement, pour 32 essais " manuels » et 20 essais "Cartier ", des teneurs en matière sèche de $41,9(1,89)$ et $41,1(0,73)$; en matière grasse, de $20,0(1,17)$ et $19,8(1,27)$; en azote total, de $2,66(0,16)$ et 2,80 $(0,10)$; en $\mathrm{NaCl}$ de $2,10(0,22)$ et $2,34(0,30)$; en calcium, de 0,33 $(0,04)$ et $0,25(0,03)$ pour cent*.

\footnotetext{
* Les nombres entre parenthèses représentent l'écart-type de la moyenne.
} 
Dans tous les cas, ces valeurs moyennes sont comprises dans l'intervalle que l'on peut trouver sur des fromages industriels. Les mesures effectuées sur des échantillons de caillé de 24 h provenant de 6 ateliers normands donnaient les moyennes et les valeurs extrêmes suivantes : $\mathrm{pH}: 4,48(4,39-4,67)$; matière sèche $(\%): 40,8(36,8-43,6)$; $\mathrm{Ca}^{++}(\%): 0,17(0,12-0,34)$.

\section{Caractéristiques microbiologiques}

Les examens bactériologiques montraient toujours l'absence de coliformes dans $1 \mathrm{~g}$ de caillé, $6 \mathrm{~h}$ après l'emprésurage. Le lait cru contenait toujours un faible nombre de levures. Ce nombre restait presque toujours inférieur à $1 \times 10^{3}$ germes $\mathrm{g}^{-1}$ et ne dépassait qu'exceptionnellement $1.10^{4}$ germes $\mathrm{g}^{-1}$ à $l^{\prime}$ intérieur du fromage. Par contre, le nombre augmentait en surface au cours de l'affinage, mais restait toujours très inférieur à celui des fromages ensemencés avec des souches de levures sélectionnées (voir plus loin).

La flore non lactique, dénombrée sur gélose au mannitol en présence de $\mathrm{NaCl}$, était inférieure à $1.10^{3}$ germes $\mathrm{g}^{-1}$ pendant toute la durée de l'affinage, alors qu'elle atteint, dans les fromages traditionnels, $5.10^{8}$ germes $\mathrm{g}^{-1}$ en surface et $5.10^{5}$ germes $\mathrm{g}^{-1}$ à l'intérieur.

\section{Caractéristiques organoleptiques}

Les caractéristiques organoleptiques des fromages préparés à Jouy selon les protocoles décrits s'éloignaient considérablement de celles des Camemberts normands. En particulier, leur flaveur était caractérisée par une amertume intense et un manque de composantes aromatiques. Au cours de la deuxième période de l'A.T.P., une part importante du travail a consisté à déterminer les causes de l'amertume et à trouver les moyens d'y remédier.

\section{III.3. Modifications organoleptiques, rhéologiques et biochimiques apportées par des modifications d'ordres microbiologique et technologique}

\section{III.3.1. Réduction de l'amertume}

a) Rôle de la présure (pub. 4, 5)

On savait que la présure était susceptible d'engendrer, à partir des caséines, des précurseurs d'amertume, notamment dans des fromages de Cheddar et de Gouda.

Le rôle de la présure dans la formation de l'amertume des fromages expérimentaux a été étudié en faisant varier la quantité de présure résiduelle des fromages par modification du $\mathrm{pH}$ du lait à l'emprésurage ou de la quantité de présure utilisée.

Des modifications du pH d'emprésurage entre 6,05 et 6,50 (il n'est pas possible de sortir de cet intervalle sans modifier profondément les fabrications) ont fait varier la quantité de présure résiduelle de $15 \%$ (teneur augmentant avec l'abaissement du $\mathrm{pH}$ ). 
On a pu faire varier beaucoup plus cette quantité en utilisant, pour la coagulation, des doses variables de présure. Lorsque la dose de présure allait de 7,5 à $60 \mathrm{ml}$ pour 1001 de lait, la quantité d'enzyme résiduelle variait de façon pratiquement linéaire, de 136 à $5700 \mu 1 . \mathrm{kg}^{-1}$ de matière sèche (volumes de présure liquide à $574 \mathrm{mg}$ de chymosine par litre).

La protéolyse du fromage était nettement influencée par la dose de présure utilisée. Dès $48 \mathrm{~h}$, la teneur en azote soluble était plus élevée dans les fromages fabriqués avec $60 \mathrm{ml}$ de présure (NS/NT $=13,5 \%$ ) que dans ceux fabriqués avec 7,5 $\mathrm{ml}(\mathrm{NS} / \mathrm{NT}=7,2 \%)$.

En ce qui concerne l'amertume, les dégustations n'ont pas permis de mettre en évidence des différences significatives entre les fromages fabriqués avec les doses extrêmes de présure. Il est probable que l'enzyme était dénaturée lorsque le $\mathrm{pH}$ de la pâte devait être égal ou supérieur à 7,0 .

\section{b) Influence de Penicillium caseicolum (pub. 5,24)}

L'incubation des fromages en atmosphère ammoniacale durant les premiers jours de l'affinage diminue visiblement l'épaisseur finale de la couche de moisissure sans retarder son apparition. Ce moyen de contrôle de la croissance de $P$. caseicolum a été utilisé pour étudier l'influence du développement de cette moisissure sur l'intensité de l'amertume. Les fromages étaient placés dans une enceinte dont la teneur atmosphérique en $\mathrm{NH}_{3}$ était maintenue entre 5 et $15 \mathrm{ppm}$. Le $\mathrm{pH}$ de surface remontait plus rapidement que celui des fromages témoins. Cette remontée plus rapide s'observait aussi au-dessous de la surface. La protéolyse était moins intense dans les fromages traités à l'ammoniac, dont la teneur en NPN était, par exemple, égale aux $2 / 3$ de celle des témoins après 21 jours d'affinage. L'examen des diagrammes électrophorétiques montrait, dans les fromages traités, une protéolyse de la caséine $\beta$ par l'aspartyl protéase du Penicillium beaucoup moins intense que dans les fromages témoins, alors que l'activité de la métalloprotéase restait du même ordre. L'amertume des fromages témoins était toujours très supérieure à celle des fromages traités, quelle que soit la dose de présure utilisée. Il apparaît donc que, dans ces fromages à flore simplifiée, les peptides amers ou leurs précurseurs soient formés principalement par action de la protéase acide sur les caséines.

\section{c) Influence de Geotrichum candidum}

Il a été montré durant l'A.T.P. qu'un ensemencement du lait en Penicillium plus Geotrichum réduisait considérablement l'amertume des fromages par rapport à celle des témoins ayant été inoculés avec du Penicillium seul.

L'ensemencement en Geotrichum entraînait un développement moins dense du Penicillium. Les examens électrophorétiques des 
zones externes des fromages révélaient des différences nettes entre témoins et essais. La dégradation des caséines $\alpha_{s_{1}}$ et $\beta$ se produisait nettement plus rapidement dans les témoins. Il apparaît que la protéase acide du Penicillium soit moins active en présence de Geotrichum comme en présence d'ammoniac. Toutefois, après 28 jours d'affinage, l'action de la protéase acide semble plus importante en présence de Geotrichum qu'en présence d'ammoniac.

Nous reparlerons plus loin des travaux qui ont été réalisés pour contrôler la croissance du Geotrichum.

\section{III.3.2. Influence de la flore sur d'autres caractéristiques organo- leptiques}

Certains groupes de micro-organismes présents naturellement en quantités importantes dans les Camemberts traditionnels de lait cru ont été ajoutés dans les fabrications «standard " mises au point. Leur influence sur les qualités organoleptiques des fromages était déterminée par analyse sensorielle effectuée avec le jury spécialement entraîné. Parallèlement, les conditions d'implantation de ces microorganismes dans les fromages ont été étudiées ainsi que certaines modifications des caractéristiques des fromages.

- Influence des levures : les levures se développent précocement dans le Camembert traditionnel. Des essais d'addition de souches représentatives des principaux groupes de levures isolées par le Laboratoire de Technologie de Grignon ont été effectués. Bien que ces levures se soient multipliées abondamment, surtout en surface, elles ont peu influencé les caractéristiques organoleptiques en présence seulement de bactéries lactiques et de Penicillium. L'effet limité qui a été observé concerne essentiellement une seule espèce.

- Effet de Geotrichum candidum (pub. 16) : parmi les souches étudiées, on peut distinguer deux groupes à partir des résultats obtenus. Celles du premier groupe diminuent l'amertume mais ne modifient pas ou presque pas l'aspect du fromage. Celles du second diminuent également l'amertume mais entraînent de plus la formation d'un arôme alliacé et d'un goût rappelant ceux du fromage normand traditionnel de lait cru. L'aspect du fromage se rapproche également de celui de ceux-ci. La croissance du Penicillium est nettement moins abondante, et la croûte est moins blanche, moins lisse et plus fripée, sans qu'on observe de défaut caractérisé. Ceci montre que l'utilisation judicieuse de ce micro-organisme pourrait permettre d'améliorer très nettement la qualité des fromages industriels de lait pasteurisé et de régulariser celle des Camemberts traditionnels de lait cru en remplaçant l'ensemencement spontané, toujours aléatoire, par un ensemencement bien choisi.

Dans cette optique, l'influence des facteurs qui conditionnent le développement de Geotrichum dans les fromages a été étudiée. Nous avons en particulier précisé le rôle déterminant de la concen- 
tration en sel et des conditions de salage sur la croissance de ce micro-organisme, sur l'apparition des qualités organoleptiques caractéristiques et sur les risques de défaut de surface.

III.3.3. Influence du pH sur les propriétés rhéologiques et physicochimiques du caillé

Penicillium caseicolum est bien connu pour les modifications de texture et l'apparition d'arôme que son développement entraîne lors de l'affinage du Camembert. Ces transformations sont généralement attribuées à l'importance de ses activités protéolytiques et lipolytiques. Cependant, le Penicillium provoque également une remontée du $\mathrm{pH}$ au sein du caillé. Celle-ci est due, d'une part à la consommation par la moisissure de l'acide lactique produit par les bactéries lactiques, et d'autre part à la formation d'ammoniac comme produit azoté final de la dégradation des protéines sous l'action de désaminases. L'importance de cette « désacidification » sur certains phénomènes qui accompagnent la maturation n'a pratiquement pas été étudiée. Elle est pourtant susceptible de modifier l'activité des protéases dans le caillé et de participer, par le biais de modifications physico-chimiques, au changement de texture. Le L.B.T.L. a tenté de déterminer quelle était l'influence du $\mathrm{pH}$ sur la texture du fromage et sur l'activité protéolytique des bactéries lactiques et de la plasmine. De son côté, le L.R.T.L. de Rennes s'est intéressé aux migrations de minéraux dans le fromage en relation avec les modifications de $\mathrm{pH}$ provoquées par le Penicillium.

\section{III.3.3.1. Travail réalisé par le L.B.T.L. (pub. 7)}

Ce laboratoire a tenté de déterminer quelle était l'influence du $\mathrm{pH}$ sur la texture du Camembert et sur l'activité protéolytique des bactéries lactiques et de la plasmine.

a) Pour l'étude rhéologique des fromages, des augmentations de $\mathrm{pH}$ au sein de fromages âgés de $48 \mathrm{~h}$ à 1 semaine ont été recréées artificiellement. Elles étaient obtenues par incubation des fromages dans une enceinte pendant 1 à $2 \mathrm{~h}$ en présence de solutions d'ammoniac. Au bout de $24 \mathrm{~h}$ d'équilibrage au froid, un gradient de $\mathrm{pH}$ décroissant, comparable à celui observé dans les fromages traditionnels, s'établissait de l'extérieur vers l'intérieur du fromage.

Des modifications de texture très importantes étaient observées dans les fromages dès la fin des traitements à l'ammoniac : une zone molle à l'extérieur, une zone dure à l'intérieur. Elles ont été appréciées par pénétrométrie à l'aide d'un appareil INSTRON. Sur chaque tranche de fromage (coupé selon un diamètre), 18 tests de pénétrométrie étaient effectués à l'aide d'une lame. Le travail mesuré lors de la pénétration était enregistré sur un mini-ordinateur et le $\mathrm{pH}$ était mesuré à l'endroit de chaque test. On obtenait des corrélations linéaires entre les $\mathrm{pH}$ et les logarithmes des travaux mesurés 
pour chaque test pour des fromages dont le gradient s'étalait sur deux unités $\mathrm{pH}$. Cependant, par des mesures complémentaires, on observait que les variations de $\mathrm{pH}$ dans un même fromage s'accompagnaient automatiquement de migrations d'eau et de calcium : la partie interne, plus dure, s'appauvrissait en eau et en calcium tandis que la partie externe, plus molle, s'enrichissait. On a pu observer avec des fromages affinés traditionnels des phénomènes similaires bien que moins accentués : la couche externe de ces fromages semble généralement plus riche en eau que la couche interne. On est donc amené à penser que le $\mathrm{pH}$ agit sur la texture en modifiant les interactions électrostatiques concernant les protéines et les teneurs en eau et en calcium de la partie externe des fromages.

b) Dans une deuxième partie, l'influence de l'augmentation du $\mathrm{pH}$ sur l'activité protéolytique de la plasmine a été étudiée. Pour ce faire, des lamelles de $3 \mathrm{~mm}$ d'épaisseur parallèles à la surface ont été découpées dans les caillés expérimentaux dans lesquels s'était établi un gradient de $\mathrm{pH}$. Ces lamelles avaient donc toutes un $\mathrm{pH}$ différent. Elles étaient traitées à la pimaricine (antifongique) et à l'acétate de thallium (bactériostatique inhibant le développement des bactéries gram $^{+}$) et enveloppées séparément dans des sacs plastiques. On avait ainsi remonté artificiellement et à différentes valeurs le $\mathrm{pH}$ des fromages et éliminé les flores fongiques et bactériennes gram+.

Au bout de 4, 8, 12 et 15 jours, les caséines étaient extraites des différents échantillons et séparées par électrophorèse d'acrylamideagarose et par électrophorèse en acrylamide-SDS. Ainsi, la protéolyse pouvait être suivie sur 15 jours, pour des échantillons de $\mathrm{pH}$ différents. On a observé une dégradation de la caséine $\beta$ au profit des caséines $\gamma$ pour les échantillons dont les $\mathrm{pH}$ étaient supérieurs à 6 . Cette dégradation de la caséine $\beta$ est typique de la plasmine dont le $\mathrm{pH}$ optimum d'activité est de 8 . On peut donc penser qu'au cours de l'affinage des Camemberts, on assiste à une augmentation de l'activité de la plasmine avec la remontée de $\mathrm{pH}$ provoquée par le Penicillium.

\section{III.3.3.2. Travail réalisé par le L.R.T.L. de Rennes (pub. 6 et 18)}

On savait qu'il se produisait une migration importante du calcium et du phosphate vers l'extérieur des pâtes molles au cours de l'implantation de la moisissure de surface. Elle avait été attribuée au «pouvoir séquestrant » du Penicillium.

Le L.R.T.L. a confirmé, pour des fromages de type Camembert, que le calcium et le phosphate migrent effectivement de l'intérieur vers la croûte où les teneurs en ces deux éléments peuvent respectivement dépasser 17 et $9 \mathrm{~g} / \mathrm{kg}$. De plus, il a montré que la migration de ces minéraux coïncidait avec le développement du Penicillium, mais était, en fait, due à l'augmentation de $\mathrm{pH}$ provoquée par la moisissure. En effet, des fromages pulvérisés avec une solution anti- 
fongique et placés en atmosphère ammoniacale, avec par conséquent, un gradient de $\mathrm{pH}$ d'origine chimique et non pas biologique, subissaient une migration minérale analogue à celle observée dans les fromages avec Penicillium. Il semble que le $\mathrm{pH}$ alcalin de surface entraîne la formation de phosphate tricalcique insoluble, ce qui conduirait à la migration vers la surface des phosphates solubles renfermés à l'intérieur plus acide du caillé. L'addition de phosphate disodique aux sels de saumure permet une migration plus importante du calcium.

Ces résultats sont importants au plan technologique, mais aussi au plan nutritionnel : il faut savoir que la croûte du Camembert affiné renferme environ $80 \%$ et $55 \%$ respectivement du calcium et du phosphore du fromage.

\section{III.4. Inventaire de la flore microbienne du camembert traditionnel (pub. $3,17,21$ )}

Nos connaissances sur la flore bactérienne des Camemberts fabriqués avec du lait cru étaient anciennes (Lenoir, 1963) et ne portaient que sur une seule fabrication. Nous avons donc fait une nouvelle étude de cette flore en suivant les fabrications de 5 usines à chaque saison. Le lait de fabrication et le fromage à divers stades de son affinage ont été examinés. Un premier inventaire de la flore bactérienne dominante des fromages a été dressé tandis que les problèmes méthodologiques liés à une telle étude et les solutions proposées ont fait l'objet d'une thèse de docteur-ingénieur. On a pu ainsi étudier avec plus de précision l'évolution de la flore bactérienne dominante et sous-dominante du lait au cours de sa maturation et de la fabrication du fromage ainsi que l'évolution de la flore spécifique de ce dernier (notamment celle des bactéries identifiables à $B$. linens), au cours de son affinage. Parallèlement, les fromages affinés ont fait l'objet d'une analyse sensorielle. De grandes différences dans la nature de la flore d'affinage ont été trouvées entre usines mais pas d'une saison à l'autre, pour une même usine. Ces différences ne se traduisent cependant pas par des goûts particuliers des fromages.

La flore d'affinage a fait l'objet d'une étude taxonomique, pour l'instant limitée par la faiblesse de nos moyens dans cette discipline, et d'une évaluation de son potentiel en matière de production de composés d'arôme dans le fromage. Deux publications sont projetées sur ce dernier sujet : l'une sur la production de phényléthanol et de phénylacétaldéhyde (pub. 20), l'autre sur la production d'un composé soufré, le méthanethiol (pub. 23).

Deux publications seront consacrées à la taxonomie de la flore d'affinage, l'une aux microcoques (pub. 21), l'autre aux «ferments du rouge » (pub. 22). 


\section{III.5. Etude de la flore de surface du camembert traditionnel par microscopie électronique à balayage (pub. 19)}

En complément de l'inventaire de la flore bactérienne de surface du Camembert traditionnel, nous avons entrepris une étude de l'aspect de la surface et de son évolution en cours d'affinage par la technique de microscopie à balayage.

Les observations montrent clairement les limites et la composition de la croûte du Camembert; elle est composée de couches superposées de divers types de micro-organismes. Les images de la croûte et des coupes à fort grossissement permettent de déterminer la nature des divers groupes de micro-organismes et leur état. Il a été ainsi observé que les bactéries lactiques restent dans le caillé, que la majeure partie des levures est concentrée dans la couche profonde de la croûte, et que les microcoques et les bactéries corynéformes sont attachées à la surface du Penicillium et ne pénètrent dans la croûte que tardivement. Les moisissures occupent la part la plus importante de la croûte. Le Geotrichum, qui paraît macroscopiquement avoir une courte existence, peut cependant être observé et continuer à se développer lentement tout au long de la période d'affinage comme nous avons pu le constater dans l'étude effectuée sur fromage expérimental. Le Penicillium présente très fréquemment des formes de dégénérescence en cours d'affinage (pub. 29).

Cette technique s'est donc avérée comme un moyen complémentaire très intéressant pour l'étude de la flore microbienne de surface du fromage.

Elle devrait nous permettre de préciser le mode d'implantation des divers types de micro-organismes en fabrication expérimentale et leur état en fonction des diverses opérations de traitement des fromages en cours d'affinage.

\section{Chapitre IV : CONCLUSIONS}

On peut dire sans hésiter que les principaux objectifs de l'A.T.P. ont été atteints, et que plusieurs résultats intéressants ont été obtenus dans des prolongements imprévus du programme initial.

La Laiterie Expérimentale de Jouy effectue maintenant régulièrement des fabrications de fromages de type Camembert que l'on peut considérer comme très reproductibles, et le jury de dégustation de Jouy s'est pérennisé et travaille à la satisfaction générale. Ces deux outils, fabrication "standard » et jury, sont à la base de tout ce qui a été fait et se fait toujours. 
Paradoxalement, la mise au point de protocoles de fabrication simplifiée, dans lesquels seulement deux micro-organismes étaient impliqués (une souche de bactéries lactiques, une souche de Penicillium), a conduit à des produits de forte et constante amertume. L'origine de ce défaut a été recherchée et il a été montré tout à fait nettement que le Penicillium, quelle que soit la souche employée, en était responsable. Des remèdes très efficaces ont également été trouvés qui, en fait, semblent agir en réduisant fortement la croissance du Penicillium : il s'agit soit d'une courte période en début d'affinage dans laquelle les fromages sont placés en atmosphère ammoniacale, soit de l'adjonction d'une deuxième moisissure, Geotrichum candidum, fréquente dans les fabrications traditionnelles. Les essais réalisés avec Geotrichum ont en outre bien montré que certaines souches sont également responsables de la formation d'une flaveur alliacée favorable que l'on trouve toujours dans les Camemberts de lait cru. Dans les deux cas (ammoniac, Geotrichum) l'analyse des fromages (fractions azotées, électrophorèse) a montré que la moindre croissance du Penicillium s'accompagnait d'une protéolyse moindre dans laquelle une seule des deux principales protéases du Penicillium avait une action beaucoup moins poussée. Cette protéase acide est très certainement impliquée dans l'apparition de peptides amers.

On savait qu'une abondante flore levure se développait précocement dans le Camembert traditionnel. Les essais d'addition de différentes levures dans le cadre de l'A.T.P. n'ont pas montré d'influence significative de ces micro-organismes sur la flaveur, tout au moins dans les conditions employées.

L'influence du $\mathrm{pH}$ (augmentation naturelle due au Penicillium, ou artificielle, en atmosphère d'ammoniac) sur la texture et la répartition des minéraux a été étudiée tant à Jouy qu'à Rennes, en mettant à profit les outils analytiques mis au point. Il semble bien qu'une remontée du $\mathrm{pH}$ dans la partie externe, en l'absence de moisissures, reproduise pratiquement les effets du Penicillium sur la texture de la pâte (ramollissement) et sur la migration du phosphate et du calcium vers la périphérie du fromage. L'addition au lait de phosphate ou de calcium permet de modifier la migration des minéraux dans un sens ou dans un autre.

Parallèlement, le "modèle » que constitue le Camembert normand de lait cru a été étudié par les microbiologistes, grâce au concours du S.V.C.N., en étroite liaison avec le jury de dégustation et le groupe " arômes » de Jouy. L'inventaire et le rôle de la flore ont été abordés. Ils tendent notamment à mettre en évidence les micro-organismes responsables, seuls ou en association, des principales caractéristiques organoleptiques. Ces études ont été complétées fort utilement par des examens en microscopie électronique à balayage qui a permis de localiser les principaux groupes microbiens du fromage et de suivre leur évolution au cours de l'affinage. 


\section{Chapitre V :}

\section{LISTE DES PUBLICATIONS ET MEMOIRES RELATIFS A L'A.T.P.}

\section{V.1. Mémoires}

1. ARchieri-Haze (M. J.) (L.B.T.L.). - Hydrolyse des caséines par les endopeptidases de $P$. caseicolum. Thèse $3^{e}$ cycle. Paris VII, Paris XI, ENSIA, janvier 1981.

2. Trieu-Cuot (P.) (L.B.T.L.). - Etude des caséines et de leurs produits de dégradation par méthodes électrophorétiques. Application à l'étude de l'affinage du Camembert. Thèse $3^{e}$ cycle. Paris VII, Paris XI, ENSIA, septembre 1981.

3. ZaDi (H.) (L.M.G.A.), - Etude de la flore bactérienne non lactique des Camemberts fabriqués de façon traditionnelle. Thèse de Docteur-Ingénieur. Paris XI, 1981.

4. Du Verdier (P. E.) (L.B.T.L.), - Effet de la dose de présure et pH du lait à l'emprésurage sur la quantité de présure retenue dans le Camembert. Influence sur l'amertume et la fermeté du fromage. Mémoire de fin d'études. ENSAR, 1981.

5. AREND (C.) (L.B.T.L.). - Influence de la présure, de la souche de streptocoque et du Penicillium sur le développement de la sensation amère dans le Camembert. Mémoire de fin d'études. ENSAR, 1982.

6. Lepienne (A.) (Rennes). - Etude de la migration des éléments minéraux au cours de l'affinage des pâtes molles en atmosphère isolée. Mémoire de fin d'études. ENSAR, septembre 1982.

7. Monnet (V.) (L.B.T.L.). - Etude de l'influence du $\mathrm{pH}$ sur la texture et la protéolyse des fromages de type Camembert. D.E.A. de Sciences Alimentaires. Paris VII, Paris XI, ENSIA, septembre 1982.

\section{V.2. Publications}

8. Trieu-Cuot (P.) and Gripon (J.C.) (L.B.T.L.). - Casein hydrolysis by P. caseicolum and $P$. roqueforti proteinases : a study with IEF and 2-dimensional electrophoresis. Neth. Milk Dairy J., 35, (1981), 353-357.

9. Trieu-Cuot (P.) et Gripon (J. C.) (L.B.T.L.). - Caractérisation de l'activité des protéases au cours de l'affinage des fromages à croûte fleurie. Symp. Internat, C.N.E.R.N.A., Use of enzymes in food Technology (1982), Ed. P. Dupuy, Lavoisier, p. 293-297.

10. TRIeu-CuOT (P.) and GRIPON (J. C.) (L.B.T.L.). - A study of proteolysis during Camembert cheese ripening using IEF and 2-dimensional electrophoresis. J. Dairy Res., 49, (1982), 501-510.

11. Trieu-Cuot (P.), Archieri-Haze (M. J.) et Gripon (J. C.) (L.B.T.L.), - Etude comparative de l'action des métalloprotéases de $P$. caseicolum et $P$. roqueforti sur les caséines $\alpha_{\mathrm{s}_{1}}$ et $\beta$. Le Lait, 62, (1982), 234-249.

12. Trieu-Cút (P.), Archieri-Haze (M. J.) and Gripon (J. C.) (L.B.T.L.). - Effect of aspartyl proteinases of $P$. caseicolum and $P$. roqueforti on caseins. $J$. Dairy Res., 49, (1982), 487-500.

13. Lebars (D.), Vassal (L.) et Gripon (J. C.) (L.B.T.L.). - Méthode d'étude de la texture du Camembert par pénétrométrie. 21e Cong. Int. Lait., 1, 502.

14. Korycka-Dahl (M.), Ribadeau-Dumas (B.), Chene (N.) and Martal (J.) (L.B.T.L., Physio-Lact.). - Plasmin activity in milk. J. Dairy Sci., 66, (1983), 704-711.

15. Korycka-Dahl (M.), Vassal (L.), Ribadeau-Dumas (B.) and Mocouot (G.) (L.B.T.L.). - Studies on lipid oxidation during ripening of Camembert cheese and its impact on cheese flavor. Sci. Aliments, 3, (1983), 79-90. 
16. Mourgues (R.), Bergère (J. L.) et Vassal (L.) (L.M.L.G.A., L.B.T.L.). - Possibilité d'améliorer les qualités organoleptiques des fromages de Camembert grâce à l'utilisation de Geotrichum candidum. La Techn. Lait., 978, (1983), 11-15.

17. Richard (J.) et ZaDI (H.) (L.M.L.G.A.). - Inventaire de la flore microbienne dominante des Camemberts fabriqués avec du lait cru. Le Lait, 63, (1983), 25-42.

18. Le Graet (Y.), Lepienne (A.), Brulé (G.) et Ducruet (P.) (Rennes). - Migration du calcium et des phosphates inorganiques dans les fromages à pâte molle de type Camembert au cours de l'affinage. Le Lait, 63, (1983), 317-332.

19. Rousseau (M.) (L.M.L.G.A.). - Study of the surface flora of traditional Camembert cheese by scanning electron miscroscopy. Milchwissenchaft, 39, (1984), 129-139.

\section{V.3. Articles soumis ou prochainement soumis pour publication}

20. LeE (C. W.) and Richard (J.) (L.M.L.G.A.). - Catabolism of L-phenylananine by some microorganisms of cheese origin. J. Dairy Sci., soumis pour publication.

21. Richard (J.) (L.M.L.G.A.). - Nature des microcoques se développant à la surface des Camemberts fabriqués avec du lait cru. An. de Mic.

22. RichaRd (J.) (L.M.L.G.A.). - Phenotypic characterization and classification of orange pigmented bacteria isolated from Camembert cheese made from raw milk. Syst. and applied Mic.

23. Hemme (D.) and Richard (J.) (L.M.L.G.A.). - L-methionine utilisation and production of methane-thiol by bacteria isolated from commercial Camemberts. J. Dairy Res.

24. VASSAL (L.) et GRIPon (J. C.) (L.B.T.L.). - L'amertume des fromages de type Camembert. Rôle de la présure et de Penicillium caseicolum. Moyens de la contrôler. 